\title{
Design and Performance of VQ-Based Hybrid Digital-Analog Joint Source-Channel Codes
}

\author{
Mikael Skoglund, Member, IEEE, Nam Phamdo, Senior Member, IEEE, and Fady Alajaji, Senior Member, IEEE
}

\begin{abstract}
A joint source-channel hybrid digital-analog (HDA) vector quantization (VQ) system is presented. The main advantage of the new VQ-based HDA system is that it achieves excellent rate-distortion-capacity performance at the design signal-to-noise ratio (SNR) while maintaining a "graceful improvement" characteristic at higher SNRs. It is demonstrated that, within the HDA framework, the parameters of the system can be optimized using an iterative procedure similar to that of channel-optimized vector quantizer design. Comparisons are made with three purely digital systems and one purely analog system. It is found that, at high SNRs, the VQ-based HDA system is superior to the other investigated systems. At low SNRs, the performance of the new scheme can be improved using the optimization procedure and using soft decoding in the digital part of the system. These results demonstrate that the introduced scheme provides an attractive method for terrestrial broadcasting applications.
\end{abstract}

Index Terms-Broadcasting, hybrid digital-analog coding, joint source-channel coding, robust transmission, source coding, vector quantization (VQ).

\section{INTRODUCTION AND MOTIVATION}

C ONSIDER the problem of transmitting a Gaussian source over a Gaussian channel. According to the source-channel separation principle [2], optimal performance can be achieved by separate, or independent, design of the source and channel codes. Systems which are designed based on this principle are often referred to as tandem source-channel coding systems. Tandem systems are always designed using digital coding techniques. A fundamental problem associated with the digital tandem system in particular, is the so-called threshold effect.

The threshold effect actually involves two problematic traits. i) First, even though these systems typically perform well relative to the Shannon limit at the designed channel signal-to-noise

Manuscript received April 16, 2000; revised October 4, 2001. The work of M. Skoglund was supported in part by the Swedish Research Council for Engineering Sciences under Grant 271-99-194. The work of F. Alajaji was supported in part by the Natural Sciences and Engineering Research Council (NSERC) of Canada. The material in this paper was presented in part at the IEEE Symposium on Information Theory, Sorrento, Italy, June 2000 [1].

M. Skoglund is with the Department of Signals, Sensors and Systems, Royal Institute of Technology, SE-100 44 Stockholm, Sweden (e-mail: skoglund@s3.kth.se).

N. Phamdo was with the Department of Electrical and Computer Engineering, State University of New York, Stony Brook, NY 11794 USA. He is now with the Applied Physics Laboratory, The Johns Hopkins University, Laurel, MD 20723 USA (e-mail: nam.phamdo@jhuapl.edu).

F. Alajaji is with the Department of Mathematics and Statistics and the Department of Electrical and Computer Engineering, Queen's University, Kingston, ON K7L 3N6, Canada (e-mail: fady@ mast.queensu.ca)

Communicated by P. A. Chou, Associate Editor for Source Coding. Publisher Item Identifier S 0018-9448(02)00633-8. ratio (SNR), there usually is a drastic degradation in performance at lower SNRs. Historically, the better these systems perform at the design SNR, the more drastic is the performance degradation at lower SNRs. This problem, which is well known in the literature, is due to the quantizer sensitivity to bit errors and the total breakdown of most powerful error-correcting codes at low SNRs. The breakdown at low SNRs is not a feature of digital tandem systems only, but a problem of nonlinear communication systems in general (that is, the breakdown typically also occurs in systems based on nonlinear analog modulation formats, such as frequency or phase modulation). ii) A second and often-overlooked problem is that as the channel SNR increases, the performance of tandem systems does not improve after a certain point. We refer to this as the leveling-off effect. This effect is due to the unrecoverable quantizer distortion which limits the system performance at high SNRs. The leveling-off effect is a feature of purely digital systems and is not in general a problem in analog systems.

To address the first problem various digital joint sourcechannel coding systems have been proposed. In these systems, the designs of the source and channel codes are either combined or are well coordinated. Examples of approaches to joint source-channel coding include: a) optimal quantizer design for noisy channels [3]-[7], b) optimization of the channel codeword assignment [6], [8], c) channel codes which use unequal error protection, and d) channel codes which are designed to exploit the residual redundancy of the source encoder output to correct channel errors [9]-[11]. These traditional joint source-channel coding systems improve the system performance at low SNRs. However, they do not address the leveling-off effect which occurs at high SNRs.

In [12] and [13], various hybrid digital-analog (HDA) joint source-channel coding systems are proposed to address the leveling-off effect. The main motivation for using an HDA system is that it can asymptotically achieve the optimal performance at the design SNR (a feature advantage of digital systems) while maintaining a "graceful improvement" characteristic at high SNRs (a feature advantage of analog systems). Thus, HDA systems combine the best of both (digital and analog) worlds. In [12] and [13], the asymptotic performances of the HDA systems are obtained. In [14], an application of an HDA system to the coding of a speech signal over a noisy Gaussian channel is presented. Other methods which combine digital and analog coding techniques include [15]-[20].

In this paper, we present a vector quantization (VQ)-based HDA joint source-channel coding system. Our main objective is to design a simple (low-complexity, low-delay) system which 


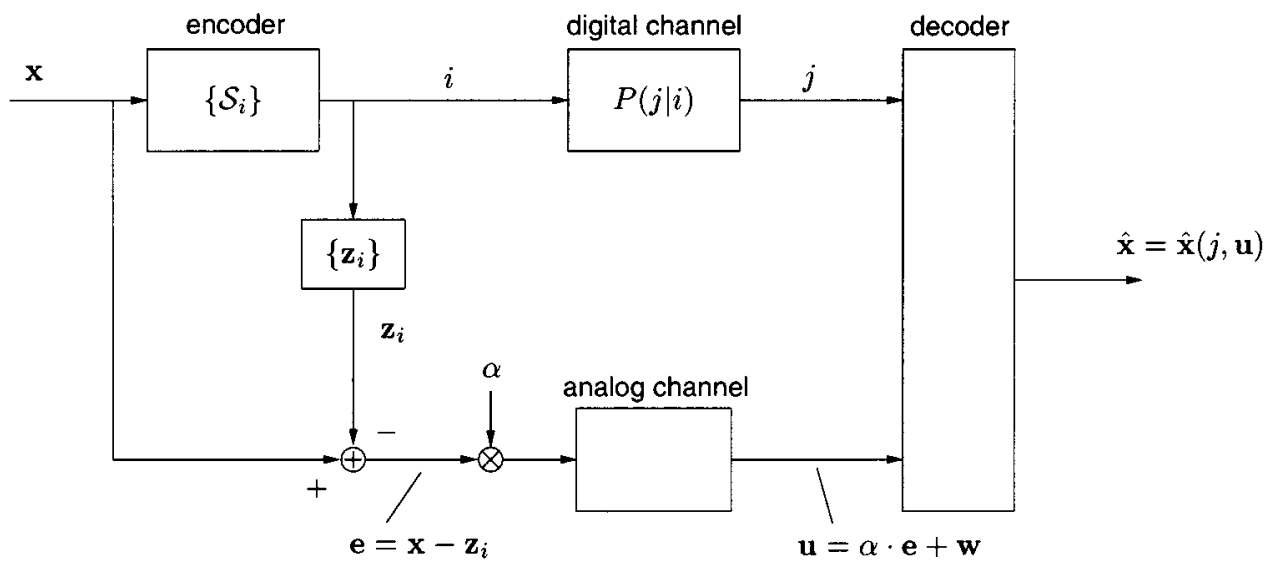

Fig. 1. Hybrid digital-analog system with joint decoding.

performs well over a wide range of channel SNRs. We assume memoryless and Gauss-Markov sources and an additive white Gaussian noise (AWGN) channel. We demonstrate that within the VQ-based HDA framework, the system can be optimized using an iterative method similar to the traditional channel-optimized VQ (COVQ) design algorithm [4], [7]. Motivated by a broadcast scenario, we then present the performance of a fixed encoder, adaptive decoder system in which the encoder is optimized for a fixed-channel SNR while the decoder adapts to the changing channel SNR. Comparisons are made with the unoptimized system, three purely digital systems, and a purely analog system. Results with soft-output demodulation in the digital part of the system are also presented.

\section{SySTEM DESCRIPTION}

In this section, we give an account for the basic assumptions made about the investigated HDA system. We begin with considering the system depicted in Fig. 1. The upper half of the figure corresponds to the "digital part" of the system, while the lower half corresponds to the "analog part." The overall purpose of the system is to convey a $d$-dimensional random source vector $^{1} \boldsymbol{X} \in \mathbb{R}^{d}$ and reproduce it as $\hat{X}$ at the receiver side, with the aim of minimizing the total distortion

$$
D \triangleq E\|\boldsymbol{X}-\hat{\boldsymbol{X}}\|^{2}
$$

or, equivalently, maximizing the signal-to-distortion ratio

$$
\mathrm{SDR} \triangleq E\|\boldsymbol{X}\|^{2} / D
$$

The individual parts of the system are described in the following.

A sample, $\boldsymbol{x}$, from the source is fed to the encoder of the system, and the encoder then produces an index $i \in\{0, \ldots, N-1\}$, with $N=2^{L}$ (where $L$ is an integer). The mapping of the encoder is specified by the encoder regions, $\left\{\mathcal{S}_{i}\right\}_{i=0}^{N-1}$, which form a partition of $\mathbb{R}^{d}$ such that when $x \in \mathcal{S}_{i}$ the encoder outputs the index $i$. Let

$$
P(i) \triangleq \operatorname{Pr}\left(\boldsymbol{X} \in \mathcal{S}_{i}\right)=\operatorname{Pr}(I=i)
$$

${ }^{1}$ Throughout, we denote random entities by capital letters, and realizations of these by the corresponding lower case letters. Bold-face symbols will be used for vectors and matrices. denote the probability that index $i$ is chosen by the encoder, and let

$$
m_{x}(i) \triangleq E\left[\boldsymbol{X} \mid \boldsymbol{X} \in \mathcal{S}_{i}\right]=E[\boldsymbol{X} \mid I=i]
$$

denote the centroid of the $i$ th encoder region. When $x \in \mathcal{S}_{i}$ the $L$ bits of the chosen index $i$ are fed to a binary symmetric channel (BSC) ${ }^{2}$ with crossover probability $q$, resulting in the output index $j$. Let

$$
P(j \mid i) \triangleq \operatorname{Pr}(J=j \mid I=i)
$$

denote the probability that index $j$ is received given that the input index to the channel was $i$. We assume that the BSC of the digital channel results from using hard decisions on a discrete-time binary-input Gaussian channel (binary phase shift keying (BPSK) modulation over an AWGN channel), with input in $\{ \pm 1\}$ and with noise variance $\sigma^{2}$ per channel use. Consequently, the transition probabilities $\{P(j \mid i)\}$ can be obtained as

$$
P(j \mid i)=q^{w(i, j)}(1-q)^{L-w(i, j)}
$$

where $w(i, j)$ denotes the Hamming distance between the binary representations of the integers $i$ and $j$, and where $q=Q(1 / \sigma)$ with

$$
Q(x)=\frac{1}{\sqrt{2 \pi}} \int_{x}^{\infty} e^{-t^{2} / 2} d t .
$$

At the transmitter, the output index $i$ of the encoder also chooses a codevector $\boldsymbol{z}_{i}$ from the encoder codebook, $\left\{\boldsymbol{z}_{i}\right\}_{i=0}^{N-1}$, and the residual vector $\boldsymbol{e}=\boldsymbol{x}-\boldsymbol{z}_{i}$ is then formed. This vector is scaled by the real constant $\alpha>0$ and transmitted over a discrete-time analog-amplitude Gaussian channel. The purpose of the scaling constant $\alpha$ is to regulate the transmission power in the analog part. The received analog vector is $\boldsymbol{u}=\alpha \cdot \boldsymbol{e}+\boldsymbol{w}$, where $\boldsymbol{w}$ is drawn according to a Gaussian distribution with zero-mean independent components of variance $\sigma^{2}$. Note that

\footnotetext{
${ }^{2}$ We assume that the digital channel can be modeled as a BSC for simplicity. Most results of this paper can, however, straightforwardly be generalized to any discrete memoryless channel model for the digital part, for example, a discrete model corresponding to a memoryless multilevel modulation scheme. To keep this fact in mind we will frequently work with a general set of transition probabilities, $\{P(j \mid i)\}$.
} 


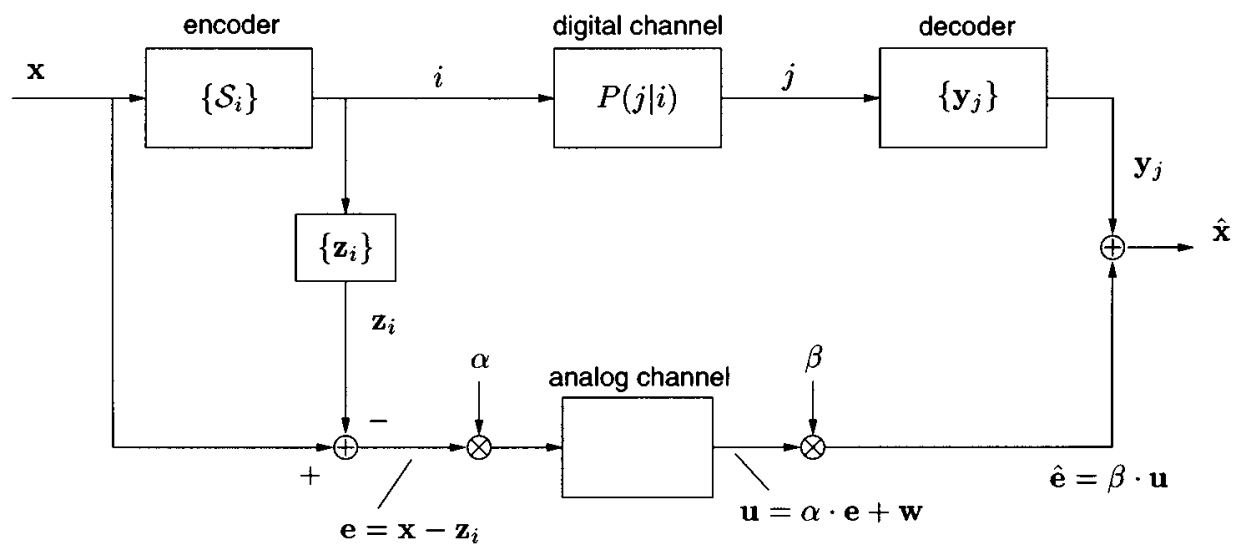

Fig. 2. Hybrid digital-analog system with simplified decoding.

we assume that the noise variance in the digital and analog parts are equal. The digital and analog parts are hence implicitly assumed to use the same underlying physical channel or two different channels with the same noise characteristics. ${ }^{3}$

In the general case, as it is illustrated in Fig. 1, the decoder of the system is a mapping $\hat{\boldsymbol{x}}=\hat{\boldsymbol{x}}(j, \boldsymbol{u})$, chosen to minimize the distortion $D$ for a given encoder, as defined by $\left\{\mathcal{S}_{i}\right\}$, a given encoder codebook, $\left\{\boldsymbol{z}_{i}\right\}$, and a fixed $\alpha$. It is straightforward to see that the optimal decoder is the minimum mean-square error (MMSE) estimator $\hat{\boldsymbol{x}}(j, \boldsymbol{u})=E[\boldsymbol{X} \mid J=j, \boldsymbol{U}=\boldsymbol{u}]$. That is,

$$
\begin{aligned}
\hat{\boldsymbol{x}}(j, \boldsymbol{u})= & \sum_{i=0}^{N-1} \int_{\mathcal{S}_{i}} \boldsymbol{x} p(\boldsymbol{x} \mid j, \boldsymbol{u}) d \boldsymbol{x} \\
= & \frac{\sum_{i=0}^{N-1} P(j \mid i) \int_{\mathcal{S}_{i}} \boldsymbol{x} p(\boldsymbol{u} \mid \boldsymbol{x}, i) p(\boldsymbol{x}) d \boldsymbol{x}}{\sum_{i=0}^{N-1} P(j \mid i) \int_{\mathcal{S}_{i}} p(\boldsymbol{u} \mid \boldsymbol{x}, i) p(\boldsymbol{x}) d \boldsymbol{x}} \\
= & \frac{\sum_{i=0}^{N-1} P(j \mid i) \int_{\mathcal{S}_{i}} \boldsymbol{x} e^{-\left(2 \sigma^{2}\right)^{-1}\left\|\boldsymbol{u}-\alpha\left(\boldsymbol{x}-\boldsymbol{z}_{i}\right)\right\|^{2}} p(\boldsymbol{x}) d \boldsymbol{x}}{\sum_{i=0}^{N-1} P(j \mid i) \int_{\mathcal{S}_{i}} e^{-\left(2 \sigma^{2}\right)^{-1}\left\|\boldsymbol{u}-\alpha\left(\boldsymbol{x}-\boldsymbol{z}_{i}\right)\right\|^{2}} p(\boldsymbol{x}) d \boldsymbol{x}}
\end{aligned}
$$

where $p(\boldsymbol{x})$ is the probability density function (pdf) of the source vector, and $p(\boldsymbol{x} \mid j, \boldsymbol{u})$ is the conditional pdf of $\boldsymbol{X}$ given $J=j$ and $\boldsymbol{U}=\boldsymbol{u}$. Furthermore, the conditional pdf $p(\boldsymbol{u} \mid \boldsymbol{x}, i)$ of $\boldsymbol{U}$ given $\boldsymbol{X}=\boldsymbol{x}$ and $\boldsymbol{X} \in \mathcal{S}_{i}$ is obtained as

$$
p(\boldsymbol{u} \mid \boldsymbol{x}, i)=\frac{1}{\left(2 \pi \sigma^{2}\right)^{d / 2}} \exp \left(-\frac{1}{2 \sigma^{2}}\left\|\boldsymbol{u}-\alpha\left(\boldsymbol{x}-\boldsymbol{z}_{i}\right)\right\|^{2}\right)
$$

since $W$ is $d$-dimensional Gaussian with independent components of variance $\sigma^{2}$. Studying (3), we see that the reproduction $\hat{\boldsymbol{x}}$ of the source vector $\boldsymbol{x}$ is based on information transmitted both via a digital and an analog channel. This fact is the key principle behind the work of this paper.

Unfortunately, the optimal joint decoder as given by (3) is very hard to implement. The reason for this is that the inte-

\footnotetext{
${ }^{3}$ The digital and analog transmissions can, for example, take place simultaneously at two different carrier frequencies, or be divided in time by alternate uses of the same carrier. However, since we assume that the noise characteristics are the same for the digital and analog parts, the most reasonable assumption about the transmission is the latter one.
}

grals in the last expression of (3) cannot, in general, be solved in closed form, and hence they must be calculated numerically or estimated for each received value of $\boldsymbol{u}$. Since one of our main objectives is to present a reasonably simple system for hybrid digital-analog coding we will not use the optimal joint decoder. Instead, having introduced the general system of Fig. 1, we turn to Fig. 2 and an approximation to the joint decoder where the digital and analog parts are decoded separately and then combined. To this end, and with reference to Fig. 2, consider the receiver side of the system with simplified decoding. In the digital part of the decoder a particular index $j$ is received, and the codevector $\boldsymbol{y}_{j}$ is produced via table lookup decoding using the decoder codebook $\left\{\boldsymbol{y}_{j}\right\}_{j=0}^{N-1}$. In the analog part, the received vector $\boldsymbol{u}$ is multiplied by a rescaling constant $\beta>0$ and then added to the codevector $\boldsymbol{y}_{j}$, resulting in an estimate of the transmitted source vector according to

$$
\hat{\boldsymbol{x}}=\beta \boldsymbol{u}+\boldsymbol{y}_{j} .
$$

We hence see that the decoder (4) combines the contributions from the digital and analog parts linearly to form a source vector estimate.

In the remaining parts of the paper we will study the system with simplified decoding as in Fig. 2, and when referring to the "HDA system" we always refer from now on to Fig. 2.

\section{PERFormance of the UnOPTIMIZED HDA SySTEM}

In this section, we investigate the performance of an unoptimized version of the VQ-based HDA system with simplified decoding, as introduced in Fig. 2. This is the most straightforward implementation of the HDA system and we refer to it as HDA-VQ. In the HDA-VQ system, the encoder regions $\left\{\mathcal{S}_{i}\right\}$ are obtained via the well-known Linde, Buzo, and Gray (LBG) design algorithm [21] for noiseless channels. Furthermore, the encoder and decoder codebooks are obtained from the centroids, that is,

$$
\boldsymbol{z}_{i}=\boldsymbol{y}_{i}=\boldsymbol{m}_{x}(i)
$$

The constant $\alpha$ is chosen to satisfy the analog channel power constraint $E\left[\alpha^{2}\left\|\boldsymbol{X}-\boldsymbol{z}_{I}\right\|^{2} / d\right]=1$ (cf., the discussion in connection with (11) below), while $\beta$ is chosen such that $\beta \cdot \boldsymbol{u}$ is 
the component-wise linear MMSE (LMMSE) estimator of the vector $\boldsymbol{E}$ based on the observation $\boldsymbol{U}=\boldsymbol{u}$, that is,

$$
\beta=\frac{1}{\alpha\left(1+\sigma^{2}\right)}
$$

(see also (13) below). Note that choosing $\beta$ according to (6) requires that $\sigma^{2}$ is known at the analog part of the receiver. Thus, the HDA-VQ system employs a "semi-adaptive" receiver where the analog part knows the noise variance while the digital part does not utilize any channel knowledge at all. Motivated by a broadcast scenario, we will, in Section V, investigate the performance of a fully adaptive receiver, where both the digital and analog parts know the channel statistics.

The performance of the HDA-VQ system for an independent and identically distributed (i.i.d.) Gaussian source and a Gauss-Markov source with correlation parameter $\rho=0.9$ is shown in Figs. 3 and 4, respectively. Here, $d=L=8$. Note that this corresponds to an overall transmission rate of $r=2$ channel uses per source sample. The LBG-VQ was designed using one million training vectors, and 500000 test vectors were employed in simulating the resulting performance. Performance is illustrated as SDR versus channel SNR, where the channel SNR in our case is obtained as SNR $=1 / \sigma^{2}$. For comparison purposes, we also present the following curves.

- The optimal performance theoretically attainable (OPTA), which is obtained by setting

$$
R(D)=r C_{\mathrm{AWGN}}
$$

where $R(D)$ is the rate-distortion function in bits per source sample of the source (under the squared-error distortion measure), $r=2$ channel uses per source sample, and $C_{\mathrm{AWGN}}$ is the channel capacity of the (continuous-input, continuous-output) AWGN channel given by

$$
\left.C_{\mathrm{AWGN}}=\frac{1}{2} \log _{2}(1+\mathrm{SNR}) \quad \text { [bits per channel use }\right] .
$$

- The resulting OPTA when the digital part of the system is restricted to the use of binary modulation, resulting in a BSC as described in Section II. This OPTA curve is obtained via

$$
R(D)=r\left(C_{\mathrm{AWGN}}+C_{\mathrm{BSC}}\right)
$$

where

$$
C_{\mathrm{BSC}}=1+q \log _{2} q+(1-q) \log _{2}(1-q)
$$

[bits per channel use],

with $q=Q(\sqrt{\mathrm{SNR}})$, is the capacity of the BSC. In Figs. 3 and 4 , the unrestricted OPTA, obtained from (7), is denoted by "OPTA ${ }_{1}$ " and the OPTA for a BSC in the digital part, obtained via (8), is denoted by "OPTA 2 ."

- A purely analog system in which each source sample is rescaled to variance one and then directly transmitted over the channel twice $(r=2)$, and where the receiver employs

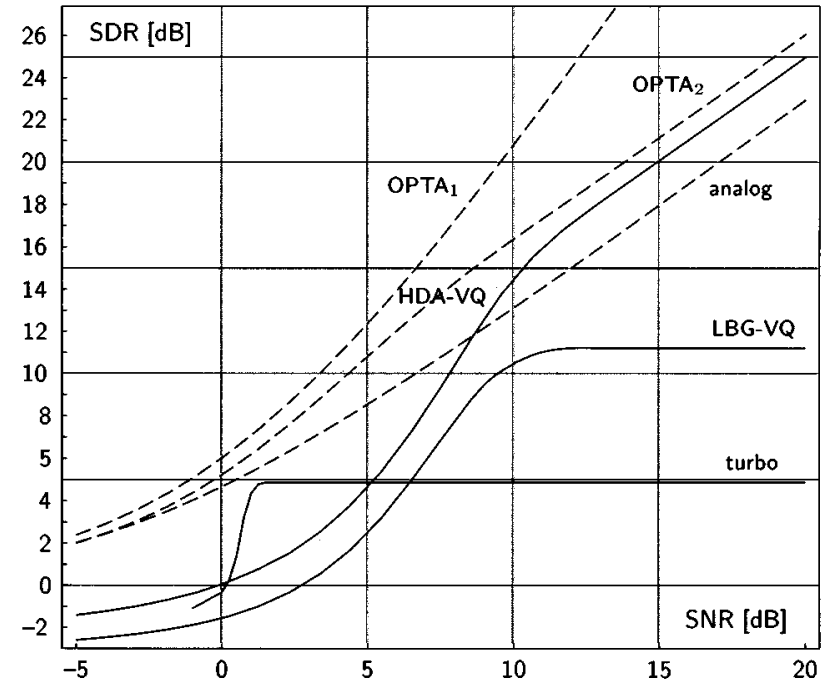

Fig. 3. Performance for unoptimized systems and an i.i.d. Gaussian source Solid lines from above at SNR $=15 \mathrm{~dB}$ : HDA-VQ, LBG-VQ $(d=8$ and rate two), LBG-VQ-turbo ( $d=8$ and overall rate two). Dashed lines from above: OPTA for an unrestricted AWGN channel in the digital part, OPTA for a BSC in the digital part, purely analog.

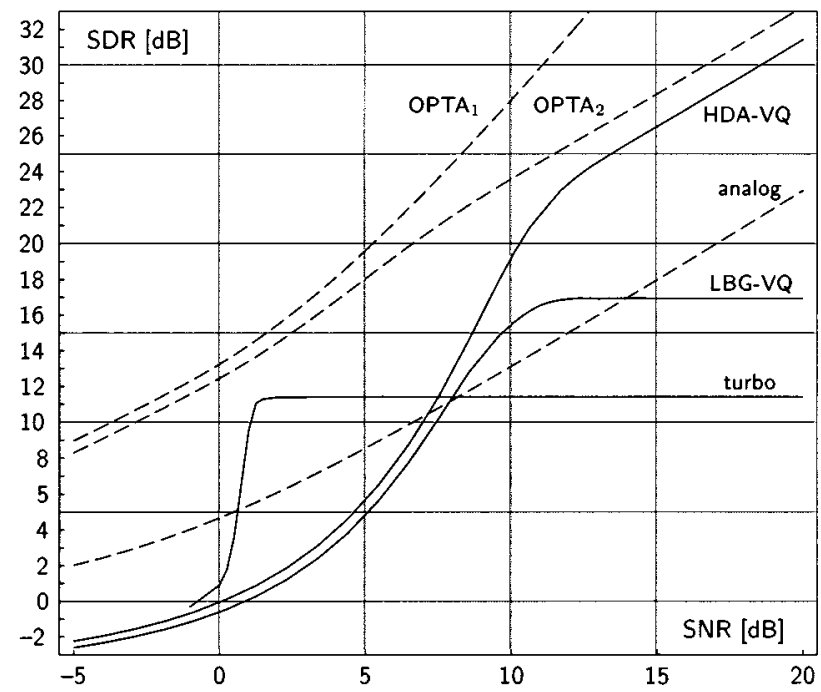

Fig. 4. Performance for unoptimized systems and a Gauss-Markov source Solid lines from above at SNR $=15 \mathrm{~dB}$ : HDA-VQ, LBG-VQ $(d=8$ and rate two), LBG-VQ-turbo ( $d=8$ and overall rate two). Dashed lines from above: OPTA for an unrestricted AWGN channel in the digital part, OPTA for a BSC in the digital part, purely analog.

an LMMSE decoder. The distortion of this scheme is given by

$$
D_{\text {analog }}=\frac{\sigma^{2}}{2+\sigma^{2}} E\|\boldsymbol{X}\|^{2} .
$$

This system is chosen for simplicity. An alternative benchmark, based on linear analog modulation, would be the system in Berger's book [22, Sec. V-B]. When viewed as a block code, Berger's system, however, has infinite block length (it is based on a concatenation of noncausal linear filters) while our HDA system uses (rather short) finite blocks. Therefore, comparing with the optimal system of [22] would be somewhat unfair to the HDA system, while comparing with (9) is still reasonable. 
- A purely digital system with no channel coding. Here, the source is quantized by an eight-dimensional, 16-bit LBG-VQ ( $d=8, L=16)$. The LBG-VQ was trained using 8 million training vectors, and simulated using 500000 test vectors. The bit assignment for the LBG-VQ is obtained from the natural splitting procedure of the LBG design algorithm [21].

- A purely digital tandem system, denoted by LBG-VQturbo, which consists of an eight-dimensional 8-bit LBGVQ $(d=L=8)$, followed by a rate- $1 / 2,(n, k)=$ $(2048,1024)$ turbo code [23] with generators $(37,21)$. Twenty iterations were applied in the turbo decoding part. Note that this system has a much higher encoding/decoding delay and complexity than the HDA-VQ system (see below). In this simulation, the LBG-VQ was designed using 250000 training vectors, and then the performance was tested using 65536 source vectors.

Observe the following. i) The strictly positive slope (slope $=1$ ) of the HDA-VQ curves at high SNRs. This is contrasted with the leveling-off effect (slope $=0$ ) of the two purely digital systems. We say that the HDA-VQ system gracefully improves at high SNRs. Note also that for high SNRs, the HDA-VQ system performs close to the OPTA for a BSC in the digital part $\left(\mathrm{OPTA}_{2}\right)$, and the performance increases with the same slope. ii) In both figures, HDA-VQ outperforms LBG-VQ at all channel SNRs. iii) For high SNRs (9 $\mathrm{dB}$ and above), HDA-VQ outperforms the other three systems. iv) The superiority of HDA-VQ over the purely analog system at high SNRs is more pronounced when the source has memory. v) At low SNRs, both purely analog and LBG-VQ-turbo outperform the HDA-VQ system. It should be noted, however, that the LBG-VQ-turbo system has a delay of 1024 samples compared with the eight-sample delay of the HDA-VQ system. Furthermore, the decoding complexity of the turbo decoder is about 3800 floating-point operations per source sample, whereas the complexity of the HDA-VQ decoder is only one operation per source sample. We will show that the performance of the HDA-VQ system at low SNRs can be improved using the optimization technique described in Section IV.

Before leaving the present section we return to observation iv) and emphasize the interesting fact that the difference in performance between the HDA-VQ system and the purely analog system appears to be constant for high SNRs $\left(\sigma^{2} \rightarrow 0\right)$. Using (9) and (5) in (14) (as derived in Section IV), it is, in fact, straightforward to show that

$$
\lim _{\sigma^{2} \rightarrow 0} \frac{D_{\text {analog }}}{D_{\mathrm{HDA}-\mathrm{VQ}}}=\frac{1}{2} \cdot \frac{E\|\boldsymbol{X}\|^{2}}{E\left\|\boldsymbol{X}-\boldsymbol{m}_{x}(I)\right\|^{2}}=\frac{1}{2} \mathrm{SDR}_{\mathrm{VQ}}
$$

where $D_{\mathrm{HDA}-\mathrm{VQ}}$ is the distortion of the HDA-VQ system in Figs. 3-4, and SDR $V$ is the SDR of the VQ part "alone" in the HDA-VQ system. Hence, the gap between the performance of the HDA-VQ and the purely analog system is indeed constant for high SNRs, since SDR ${ }_{V Q}$ does not depend on $\sigma^{2}$. Furthermore it is clear that the gap is larger for sources with memory since SDR $\mathrm{VQ}_{\mathrm{Q}}$ typically can be made to increase with increasing source correlation. This conclusion about the differ- ence in performance between the HDA system and the purely analog system can be generalized to hold also for the optimized HDA systems simulated in Section V.

\section{DESIGN OF OPTIMIZED HDA SySTEMS}

In this section, we consider an optimization technique for improving the performance of the HDA system in Fig. 2. The treatment will lead up to an algorithm that strives to minimize the distortion

$$
D=E\|X-\hat{\boldsymbol{X}}\|^{2}
$$

at a given "design SNR" $1 / \sigma^{2}$ and under a constraint on the transmitted power

$$
P_{a} \triangleq \frac{1}{d} \alpha^{2} E\left\|\boldsymbol{X}-z_{I}\right\|^{2}
$$

per channel use in the analog part. More precisely, the aim of the design will be to find encoder regions $\left\{\mathcal{S}_{i}\right\}$, encoder codevectors $\left\{\boldsymbol{z}_{i}\right\}$, decoder codevectors $\left\{\boldsymbol{y}_{j}\right\}$, and a decoder rescaling constant $\beta$, such that $D$ is minimized under the constraint that $\alpha$ is chosen such that $P_{a}=1$ is satisfied at all times, that is, the SNRs in the digital and analog parts are constrained to be equal. This power constraint is imposed for two reasons. i) Since we (implicitly) assume that the digital and analog parts of the HDA system use the same carrier on the underlying physical channel in a time-division mode, it is natural to assign the digital and analog transmission equal power. This is because a time-varying transmission power typically would entail costly requirements on the power amplifier in the transmitter. ii) Assigning equal power to both parts is motivated also by our objective to design a transmitter that is robust against variations in the channel SNR. Even if we need to specify a design SNR in the system optimization, it is still desirable to allocate equal power from the point of view of making as few additional assumptions about the channel quality as possible.

To begin our treatment of how to optimize the system we first look at the expression for the overall distortion $D$. To this end, we note that for arbitrary $\left\{\mathcal{S}_{i}\right\},\left\{\boldsymbol{z}_{i}\right\},\left\{\boldsymbol{y}_{j}\right\}$, and $\beta$, but with $\alpha$ chosen to satisfy the power constraint, the distortion can be expressed as

$$
\begin{aligned}
D= & E\|\boldsymbol{X}-\hat{\boldsymbol{X}}\|^{2}=E\left\|\boldsymbol{X}-\left(\beta \cdot \boldsymbol{U}+\boldsymbol{y}_{J}\right)\right\|^{2} \\
= & E\left\|\boldsymbol{X}-\boldsymbol{y}_{J}\right\|^{2}-2 \gamma E\left[\left(\boldsymbol{X}-\boldsymbol{z}_{I}\right)^{T}\left(\boldsymbol{X}-\boldsymbol{y}_{J}\right)\right] \\
& +E\left\|\gamma\left(\boldsymbol{X}-\boldsymbol{z}_{I}\right)+\beta \boldsymbol{W}\right\|^{2} \\
= & E\left\|\boldsymbol{X}-\boldsymbol{y}_{J}\right\|^{2}-2 \gamma E\left[\left(\boldsymbol{X}-\boldsymbol{z}_{I}\right)^{T}\left(\boldsymbol{X}-\boldsymbol{y}_{J}\right)\right]+\beta^{2} d\left(1+\sigma^{2}\right)
\end{aligned}
$$

where $\gamma \triangleq \alpha \beta$, and where we have assumed that $\alpha^{2} E\left\|\boldsymbol{X}-z_{I}\right\|^{2}=$ $d$ is satisfied. Based on this expression for the distortion, we will derive optimality criteria for all parameters of the system. In Section IV-A, we show how $\left\{\boldsymbol{z}_{i}\right\},\left\{\boldsymbol{y}_{j}\right\}$, and $\beta$ can be chosen to minimize the distortion (under the constraint $P_{a}=1$ ), for a given set of encoder regions $\left\{\mathcal{S}_{i}\right\}$. Then, in Section IV-B, we derive an expression for the optimal encoder regions, for given values of the other parameters of the system (and, again, as- 
suming that the power constraint is satisfied). In Section IV-C, we investigate the structure of the system in some asymptotic cases, for example, how the optimal encoder regions change as the noise variance in the analog part goes to zero or infinity. Finally, in Section IV-D, we use the results of Sections IV-A and $-\mathrm{B}$ to formulate an iterative design algorithm for the whole system.

\section{A. Optimizing for Fixed Encoder Regions}

Assume that $\left\{\boldsymbol{z}_{i}\right\},\left\{\boldsymbol{y}_{j}\right\}$, and $\beta$ are arbitrary, but that the set of encoder regions $\left\{\mathcal{S}_{i}\right\}$ is known and fixed. Also assume that $\alpha$ is chosen such that the power constraint is satisfied at all times. Let us first focus on how the encoder codevectors $\left\{\boldsymbol{z}_{i}\right\}$ should be chosen to minimize the distortion under these assumptions. We note that the only term in the last equality of (12) that can be influenced by changing $\left\{\boldsymbol{z}_{i}\right\}$ is the middle one. Hence, the encoder codevectors should be chosen to maximize

$$
\gamma \cdot E\left[\left(\boldsymbol{X}-\boldsymbol{z}_{I}\right)^{T}\left(\boldsymbol{X}-\boldsymbol{y}_{J}\right)\right]
$$

Note that

$$
\begin{aligned}
E & {\left[\left(\boldsymbol{X}-\boldsymbol{z}_{I}\right)^{T}\left(\boldsymbol{X}-\boldsymbol{y}_{J}\right)\right] } \\
& =E\|\boldsymbol{X}\|^{2}-\sum_{i=0}^{N-1} P(i)\left(\boldsymbol{z}_{i}^{T} \boldsymbol{m}_{x}(i)+\boldsymbol{m}_{x}(i)^{T} \boldsymbol{m}_{y}(i)-\boldsymbol{z}_{i}^{T} \boldsymbol{m}_{y}(i)\right) \\
& =E\|\boldsymbol{X}\|^{2}-E\left[\boldsymbol{X}^{T}\left(\boldsymbol{z}_{I}+m_{y}(I)\right)\right]+E\left[\boldsymbol{z}_{I}^{T} \boldsymbol{m}_{y}(I)\right] \\
& =E\left[\left(\boldsymbol{X}-\boldsymbol{z}_{I}\right)^{T}\left(\boldsymbol{X}-\boldsymbol{m}_{y}(I)\right)\right]
\end{aligned}
$$

where $\boldsymbol{m}_{y}(i) \triangleq E\left[\boldsymbol{y}_{J} \mid I=i\right]$. Applying Schwarz's inequality, we get

$$
\begin{aligned}
\gamma \cdot E[ & \left.\left(\boldsymbol{X}-\boldsymbol{z}_{I}\right)^{T}\left(\boldsymbol{X}-\boldsymbol{m}_{y}(I)\right)\right] \\
& =\beta \cdot E\left[\left(\alpha\left(\boldsymbol{X}-\boldsymbol{z}_{I}\right)\right)^{T}\left(\boldsymbol{X}-m_{y}(I)\right)\right] \\
& \leq \beta \cdot \sqrt{E\left\|\alpha\left(\boldsymbol{X}-\boldsymbol{z}_{I}\right)\right\|^{2} E\left\|\boldsymbol{X}-\boldsymbol{m}_{y}(I)\right\|^{2}} \\
& =\beta \cdot \sqrt{d \cdot E\left\|\boldsymbol{X}-\boldsymbol{m}_{y}(I)\right\|^{2}}
\end{aligned}
$$

for all $\left\{\boldsymbol{z}_{i}\right\}$. The last term does not depend on $\left\{\boldsymbol{z}_{i}\right\}$, and it can easily be verified that the inequality can be achieved with equality by letting $\boldsymbol{z}_{i}=\boldsymbol{m}_{y}(i)$. Consequently, the encoder codevectors should be chosen as $\boldsymbol{z}_{i}=m_{y}(i), i=0, \ldots, N-1$. Note that this choice of $\left\{\boldsymbol{z}_{i}\right\}$ is consistent with the result derived in [24] for a two-stage digital coding system (see [24, text following eq. (30)]). Now, letting $\boldsymbol{z}_{i}=\boldsymbol{m}_{y}(i)$, we get the distortion

$$
\begin{aligned}
D & =E\left\|\boldsymbol{X}-\boldsymbol{y}_{J}\right\|^{2}-2 \gamma E\left\|\boldsymbol{X}-\boldsymbol{m}_{y}(I)\right\|^{2}+\beta^{2} d\left(1+\sigma^{2}\right) \\
& =E\left\|\boldsymbol{X}-\boldsymbol{y}_{J}\right\|^{2}-2 \frac{\beta}{\alpha} E\left\|\alpha\left(\boldsymbol{X}-\boldsymbol{m}_{y}(I)\right)\right\|^{2}+\beta^{2} d\left(1+\sigma^{2}\right) \\
& =E\left\|\boldsymbol{X}-\boldsymbol{y}_{J}\right\|^{2}-2 \frac{\beta}{\alpha} \boldsymbol{d}+\beta^{2} d\left(1+\sigma^{2}\right) .
\end{aligned}
$$

Hence, we see that for a fixed $\left\{\mathcal{S}_{i}\right\}$ and an arbitrary $\left\{\boldsymbol{y}_{j}\right\}$, and with $\left\{\boldsymbol{z}_{i}\right\}$ chosen as $\boldsymbol{z}_{i}=\boldsymbol{m}_{y}(i)$, the optimal $\beta$ is

$$
\begin{aligned}
\beta & =\arg \min _{\beta^{\prime}}\left\{\left(\beta^{\prime}\right)^{2} d\left(1+\sigma^{2}\right)-2 \frac{\beta^{\prime}}{\alpha} d\right\} \\
& =\frac{1}{\alpha} \cdot \frac{1}{1+\sigma^{2}} .
\end{aligned}
$$

That is, since it is straightforward to check that this value of $\beta$ also minimizes $E\|\boldsymbol{E}-\beta \boldsymbol{U}\|^{2}$, we have that $\beta \boldsymbol{u}$ is the component-wise LMMSE estimator of $\boldsymbol{E}$ based on the observation $\boldsymbol{U}=\boldsymbol{u}$. When $\beta$ is set according to (13) the resulting distortion is

$$
D=E\left\|\boldsymbol{X}-\boldsymbol{y}_{J}\right\|^{2}-\frac{1}{1+\sigma^{2}} \cdot E\left\|\boldsymbol{X}-m_{y}(I)\right\|^{2} .
$$

We now consider how the decoder codevectors $\left\{\boldsymbol{y}_{j}\right\}$ should be chosen to minimize the distortion. Since (10) can be rewritten as

$$
D=E\left\|(\boldsymbol{X}-\beta \boldsymbol{U})-\boldsymbol{y}_{J}\right\|^{2}
$$

it is obvious that the codevectors that minimize $D$ are obtained by letting $\boldsymbol{y}_{j}$ represent the MMSE estimator $E[\boldsymbol{X}-\beta \boldsymbol{U} \mid J=j]$ of the vector $\boldsymbol{X}-\beta \boldsymbol{U}$ based on the observation $J=j$. That is, $\boldsymbol{y}_{j}$ should be chosen as

$$
\begin{aligned}
\boldsymbol{y}_{j} & =E\left[\boldsymbol{X}-\beta\left(\alpha\left(\boldsymbol{X}-\boldsymbol{z}_{I}\right)+\boldsymbol{W}\right) \mid J=j\right] \\
& =(1-\gamma) \tilde{\boldsymbol{x}}(j)+\gamma \tilde{\boldsymbol{z}}(j),
\end{aligned}
$$

where

$$
\tilde{\boldsymbol{x}}(j) \triangleq E[\boldsymbol{X} \mid J=j] \quad \text { and } \quad \tilde{\boldsymbol{z}}(j) \triangleq E\left[\boldsymbol{z}_{I} \mid J=j\right] .
$$

Hence, to summarize, we have so far derived that for a given set $\left\{\mathcal{S}_{i}\right\}$ the encoder codevectors should be chosen as $\boldsymbol{z}_{i}=$ $E\left[\boldsymbol{y}_{J} \mid I=i\right]$, the rescaling constant $\beta$ as in (13), and the decoder codevectors as in (15) and (16). We note that for a given $\alpha$, the optimal value for $\beta$ depends only on the channel SNR. Consequently, $\beta$ can be chosen independently of $\left\{\boldsymbol{z}_{i}\right\}$ and $\left\{\boldsymbol{y}_{j}\right\}$. However, the expressions for $\boldsymbol{z}_{i}$ and $\boldsymbol{y}_{j}$ are not independent. In fact, these expressions can be combined so that the encoder and decoder codevectors may be obtained jointly. Such a result is presented in the following paragraph.

Letting

$$
P(j) \triangleq \operatorname{Pr}(J=j)=\sum_{i} P(j \mid i) P(i)
$$

and

$$
P(i \mid j) \triangleq P r(I=i \mid J=j)=P(j \mid i) P(i) / P(j)
$$

the expressions for $\boldsymbol{z}_{i}$ and $\boldsymbol{y}_{j}$ can be rewritten as

$$
\begin{aligned}
\boldsymbol{z}_{i} & =\sum_{j=0}^{N-1} P(j \mid i) \boldsymbol{y}_{j} \\
\boldsymbol{y}_{j} & =(1-\gamma) \tilde{\boldsymbol{x}}(j)+\gamma \cdot \sum_{i=0}^{N-1} P(i \mid j) \boldsymbol{z}_{i}
\end{aligned}
$$

with

$$
\begin{aligned}
\tilde{\boldsymbol{x}}(j) & =E[\boldsymbol{X} \mid J=j]=\sum_{i=0}^{N-1} E[\boldsymbol{X} \mid I=i, J=j] P(i \mid j) \\
& =\sum_{i=0}^{N-1} \boldsymbol{m}_{x}(i) P(i \mid j) .
\end{aligned}
$$

Now we note that (17) can be used in (18) to give the $N$ simultaneous equations

$$
\begin{aligned}
\boldsymbol{y}_{k}= & (1-\gamma) \tilde{\boldsymbol{x}}(k)+\gamma \sum_{i=0}^{N-1} \sum_{j=0}^{N-1} \operatorname{Pr}(I=i \mid J=k) \\
& \cdot \operatorname{Pr}(J=j \mid I=i) \boldsymbol{y}_{j}, \quad k=0, \ldots, N-1
\end{aligned}
$$


for the decoder codevectors $\left\{\boldsymbol{y}_{j}\right\}$. These can be put into matrix form by defining the matrices

$$
\boldsymbol{Y} \triangleq\left[\boldsymbol{y}_{0} \cdots \boldsymbol{y}_{N-1}\right] \quad \text { and } \quad \tilde{\boldsymbol{X}} \triangleq[\tilde{\boldsymbol{x}}(0) \cdots \tilde{\boldsymbol{x}}(N-1)]
$$

and the $N \times N$ matrix $\boldsymbol{F}$ with elements

$$
\begin{aligned}
(\boldsymbol{F})_{j k}=\sum_{i=0}^{N-1} \operatorname{Pr}(I=i \mid J=k) \operatorname{Pr}(J=j \mid I=i), \\
j=0, \ldots, N-1, k=0, \ldots, N-1
\end{aligned}
$$

and then observing that (20) is equivalent to the matrix equation

$$
\boldsymbol{Y}\left(\boldsymbol{I}_{N}-\gamma \boldsymbol{F}\right)=(1-\gamma) \tilde{\boldsymbol{X}}
$$

where $\boldsymbol{I}_{N}$ is the $N \times N$ identity matrix. It is important to note, in connection with (22), that the matrix $\boldsymbol{I}_{N}-\gamma \boldsymbol{F}$ is invertible for all $0 \leq \gamma<1$ (a proof of this statement is provided in the Appendix). Consequently, since with $\beta$ chosen according to (13), and with $0<\sigma^{2}<\infty$, we have that $0<\gamma<1$, we can safely assume that a unique $Y$ can always be found by solving (22). Then, with $\left\{\boldsymbol{y}_{j}\right\}$ extracted from the resulting $\boldsymbol{Y}$ the encoder codevectors are obtained using (17).

As an alternative to using (22) and (17) to solve for $\left\{\boldsymbol{y}_{j}\right\}$ and $\left\{\boldsymbol{z}_{\boldsymbol{i}}\right\}$ jointly, an iterative approach may be used. Such an approach can be initialized, for example, by letting $\boldsymbol{z}_{i}=\boldsymbol{m}_{x}(i)$. Then $\left\{\boldsymbol{y}_{j}\right\}$ can be computed using (18), and for this set of decoder codevectors $\left\{\boldsymbol{z}_{i}\right\}$ can be computed according to (17). Then a new decoder codebook can be obtained using (18) and a new $\left\{\boldsymbol{z}_{i}\right\}$ using (17), and so on, until the two codebooks have reached stable values. Two situations when using this iterative approach may be preferred over the direct approach of solving (22): i) when $N$ is (very) large, so that the matrix $\boldsymbol{I}_{N}-\gamma \boldsymbol{F}$ becomes hard to compute and store, and solving the equation system becomes too complex and ii) when $\gamma$ is close to one (corresponding to $\sigma^{2} \rightarrow 0$ ) so that $\left(\boldsymbol{I}_{N}-\gamma \boldsymbol{F}\right)$ becomes ill-conditioned.

\section{B. Optimal Encoder Regions}

Here we assume, instead, that an arbitrary $\left\{\boldsymbol{y}_{j}\right\}$ is given, and that for this given $\left\{\boldsymbol{y}_{j}\right\}$ the encoder codevectors $\left\{\boldsymbol{z}_{i}\right\}$ are chosen as $\boldsymbol{z}_{i}=m_{y}(i)=E\left[\boldsymbol{y}_{J} \mid I=i\right]$ and the rescaling constant as $\beta=\alpha^{-1} /\left(1+\sigma^{2}\right)$, under the assumption that $\alpha$ is chosen such that $\alpha^{2} \cdot E\left\|\boldsymbol{X}-\boldsymbol{z}_{I}\right\|^{2}=d$ is satisfied at all times. Then, with $\gamma=\alpha \beta=1 /\left(1+\sigma^{2}\right)$ and utilizing the fact that $E\left[\boldsymbol{X}^{T} \boldsymbol{y}_{J}\right]=E\left[\boldsymbol{X}^{T} \boldsymbol{m}_{y}(I)\right]$, an expression for the resulting distortion can obtained from (14) as

$$
\begin{aligned}
D= & E\left\|\boldsymbol{X}-\boldsymbol{y}_{J}\right\|^{2}-\gamma E\left\|\boldsymbol{X}-\boldsymbol{m}_{y}(I)\right\|^{2} \\
= & (1-\gamma) E\|\boldsymbol{X}\|^{2}-2\left(E\left[\boldsymbol{X}^{T} \boldsymbol{y}_{J}\right]-\gamma E\left[\boldsymbol{X}^{T} \boldsymbol{m}_{y}(I)\right]\right) \\
& +E\left\|\boldsymbol{y}_{J}\right\|^{2}-\gamma E\left\|\boldsymbol{m}_{y}(I)\right\|^{2} \\
= & (1-\gamma) E\left\|\boldsymbol{X}-\boldsymbol{m}_{y}(I)\right\|^{2}+E\left\|\boldsymbol{y}_{J}\right\|^{2}-E\left\|\boldsymbol{m}_{y}(I)\right\|^{2} \\
= & \sum_{i=0}^{N-1} \int_{\mathcal{S}_{i}}\left\{(1-\gamma)\left\|\boldsymbol{x}-\boldsymbol{m}_{y}(i)\right\|^{2}+g_{i}\right\} p(\boldsymbol{x}) d \boldsymbol{x}
\end{aligned}
$$

where

$$
\begin{aligned}
g_{i} & \triangleq E\left[\left\|\boldsymbol{y}_{J}\right\|^{2} \mid I=i\right]-\left\|m_{y}(i)\right\|^{2} \\
& =\sum_{j=0}^{N-1} P(j \mid i)\left\|\boldsymbol{y}_{j}\right\|^{2}-\left\|m_{y}(i)\right\|^{2} .
\end{aligned}
$$

Consequently, since $p(\boldsymbol{x})$ is nonnegative, the $i$ th encoder region $\mathcal{S}_{i}$ should be assigned those $\boldsymbol{x}$ that minimize the term within brackets in the last equality of (23). That is, the optimal encoder regions are

$$
\begin{aligned}
\mathcal{S}_{i}=\left\{\boldsymbol{x} \in \mathbb{R}^{d}:(1-\gamma)\left\|\boldsymbol{x}-\boldsymbol{m}_{y}(i)\right\|^{2}+g_{i}\right. \\
\left.\leq(1-\gamma)\left\|\boldsymbol{x}-\boldsymbol{m}_{y}(k)\right\|^{2}+g_{k}, \forall k\right\} .
\end{aligned}
$$

The expression given in (25) can be put into a form that allows for some further interpretation. Let $\overline{\boldsymbol{x}}=\left[\boldsymbol{x}^{T} 0\right]^{T}$, that is, the vector in $\mathbb{R}^{d+1}$ defined by augmenting the source vector $\boldsymbol{x}$ with a zero, and

$$
\overline{\boldsymbol{m}}_{y}(i)=\left[\boldsymbol{m}_{y}^{T}(i) \sqrt{g_{i} /(1-\gamma)}\right]^{T}
$$

(assuming $0 \leq \gamma<1$ ), that is, the vector in $\mathbb{R}^{d+1}$ obtained by augmenting $\boldsymbol{m}_{y}(i)$ with the scalar $\sqrt{g_{i} /(1-\gamma)}$ in the $(d+1)$ th position. Then (25) can be rewritten as

$$
\mathcal{S}_{i}=\left\{\boldsymbol{x} \in \mathbb{R}^{d}:\left\|\overline{\boldsymbol{x}}-\overline{\boldsymbol{m}}_{y}(i)\right\|^{2} \leq\left\|\overline{\boldsymbol{x}}-\overline{\boldsymbol{m}}_{y}(k)\right\|^{2}, \forall k\right\} .
$$

That is, letting $\Omega_{i}$ be the $i$ th region in the Voronoi partition of $\mathbb{R}^{d+1}$ defined by the vectors $\left\{\overline{\boldsymbol{m}}_{y}(i)\right\}$, the $i$ th $d$-dimensional optimal region $\mathcal{S}_{i}$ is obtained as the intersection ${ }^{4}$ of $\Omega_{i}$ with the hyperplane $\Pi=\left\{\bar{x}=\left[x^{T} 0\right]^{T}: \boldsymbol{x} \in \mathbb{R}^{d}\right\}$. For some indexes $i$, it may be the case that this intersection is empty. Hence, these indexes can never be chosen by the encoder and are, thus, totally redundant. The redundancy in the transmitted data induced by this phenomenon is utilized by the decoder for error protection (cf., [7], [25], [26] for similar results on VQ over binary discrete channels, and binary-input soft-output channels). In the following subsection, we utilize (26) to provide some interpretations of how the optimal regions vary as a function of the transition probabilities in the digital part and the noise variance in the analog part.

\section{Some Special Cases and Interpretations}

Above, we have assumed that the noise variance in the digital and analog parts are equal. That is, the value of $\sigma^{2}$ influences both the variance of the noise in the analog channel and the transition probabilities $P(j \mid i)$ of the digital channel. In this subsection, we temporarily let go of this assumption with the purpose of investigating some special cases in terms of asymptotic values for $\sigma^{2}$ and $P(j \mid i)$. To this end, let $\sigma^{2}$ denote the noise in the analog part (only), and assume that $\sigma^{2}$ and $P(j \mid i)$ are independent. Also assume that $\left\{\boldsymbol{z}_{i}\right\},\left\{\boldsymbol{y}_{j}\right\}$, and $\gamma=\alpha \beta$ are chosen according to the treatment in Section IV-A and assume, furthermore, that the encoder regions $\left\{\mathcal{S}_{i}\right\}$ satisfy (25). Then consider the following situations.

Arbitrary, but fixed, transition probabilities $P(j \mid i)$ and the following.

- Low noise level in the analog part, $\sigma^{2} \rightarrow 0$ : Since $\sigma^{2} \rightarrow 0$ implies $\gamma \rightarrow 1$ the last component of $\overline{\boldsymbol{m}}_{y}(i)$, in (26), goes to infinity. Hence, as a result, the vectors $\bar{m}_{y}(i)$, in the augmented source space, will move away from the hyperplane $\Pi$. Consequently, more and more regions $\mathcal{S}_{i}$ will

\footnotetext{
${ }^{4}$ Strictly speaking, this intersection is still a $(d+1)$-dimensional set (in the sense that its elements are members of $\mathbb{R}^{d+1}$ ). A more rigorous definition of the mapping from $\Omega_{i}$ to $\mathcal{S}_{i}$ would be to say that $\mathcal{S}_{i}$ is the projection of the intersection $\Pi \cap \Omega_{i}$ onto $\mathbb{R}^{d}$ obtained by leaving out the last coordinate (which is 0 ) in the elements of $\Pi \cap \Omega_{i}$.
} 
become empty, and the redundancy content in the transmitted digital data will increase until, finally, there is only one nonempty region left. This fact can be interpreted by noting that since the analog channel is of increasingly good quality, the digital part can "afford" to use more and more redundancy to protects its data. Now consider instead the optimal codevectors. We show in the Appendix that as $\gamma \rightarrow 1$ the solution $\boldsymbol{Y}$ of (22) approaches a matrix with all columns equal. ${ }^{5}$ That is, all decoder codevectors $\left\{\boldsymbol{y}_{j}\right\}$ will become equal, say $\boldsymbol{y}_{j}=\boldsymbol{y}_{0} \forall j$. Furthermore, by studying (17) we see that this will also imply $\boldsymbol{z}_{i}=\boldsymbol{y}_{0} \forall i$. Regarding $\left\{\boldsymbol{y}_{j}\right\}$ the fact that the codevectors become increasingly equal is reasonable since, as we have noted, the redundancy content in the transmitted digital data increases (equal codevectors can be interpreted as error-correction coding [25]). Moreover, as the analog part becomes noiseless we have $\hat{\boldsymbol{e}} \rightarrow \boldsymbol{x}-\boldsymbol{z}_{i}$ (cf., Fig. 2), and since $\boldsymbol{z}_{i} \rightarrow \boldsymbol{y}_{j} \rightarrow \boldsymbol{y}_{0}$ this gives $\hat{\boldsymbol{x}} \rightarrow \boldsymbol{y}_{0}+\left(\boldsymbol{x}-\boldsymbol{y}_{0}\right)=\boldsymbol{x}$, so the fact that $\boldsymbol{z}_{i} \rightarrow \boldsymbol{y}_{0}$ is also motivated.

- High noise level in the analog part, $\sigma^{2} \rightarrow \infty$ : Assuming $0<\alpha<\infty$ this implies $\beta \rightarrow 0$. That is, the information stemming from the analog part will not be used by the receiver (which is reasonable, since the quality of this information will be increasingly bad as $\sigma^{2} \rightarrow \infty$ ). Moreover, since $\gamma \rightarrow 0$ the equations describing the digital system, e.g., (15) and (25), will converge to the corresponding expressions for a purely digital system (cf., e.g., [7] and [25]). Hence, the digital part will depend only on the transition probabilities $\{P(j \mid i)\}$, independently of the analog part, and the analog part will be completely "turned off."

Arbitrary, but fixed, $0<\sigma^{2}<\infty$ and the following.

- Low noise level in the digital part: That is

$$
P(j \mid i) \rightarrow 1, \quad \text { if } j=i \text {; and } P(j \mid i) \rightarrow 0, \quad \text { otherwise. }
$$

First we note that as the digital channel becomes noiseless, the matrix $\boldsymbol{F}$ in (22) approaches an identity matrix. Hence, noting that $\gamma \neq 1$ (since we assume $0<\sigma^{2}<\infty$ ) the solution $\boldsymbol{Y}$ of (22) will, in the limit, be equal to the matrix $\tilde{\boldsymbol{X}}$, that is, $\boldsymbol{y}_{j}=\tilde{\boldsymbol{x}}(j)$. Furthermore, from (19), we have that $\tilde{\boldsymbol{x}}(j) \rightarrow \boldsymbol{m}_{x}(j)=E[\boldsymbol{X} \mid I=j]$, and studying (17) we see that $\boldsymbol{z}_{i} \rightarrow \boldsymbol{y}_{i}$. Hence, in the limit it holds that the vectors $\left\{\boldsymbol{y}_{j}\right\},\left\{\boldsymbol{z}_{i}\right\}$, and $\left\{\boldsymbol{m}_{x}(i)\right\}$ are all equal. Now, turning to the optimal encoder regions we first note that as the digital channel becomes noiseless, we have that $\boldsymbol{m}_{y}(i) \rightarrow \boldsymbol{m}_{x}(i)$. Hence, studying (24) we see that $g_{i} \rightarrow\left\|\boldsymbol{m}_{x}(i)\right\|^{2}-\left\|\boldsymbol{m}_{x}(i)\right\|^{2}=0$. Consequently, from (25) (and, again, noting that $\gamma \neq 1$ ), we have that the optimal regions $\left\{\mathcal{S}_{i}\right\}$ will approach the Voronoi regions defined by the vectors $\left\{m_{x}(i)\right\}$. Hence, to summarize, since the codevectors $\left\{\boldsymbol{y}_{j}\right\}$ approach the encoder centroids $\left\{\boldsymbol{m}_{x}(i)\right\}$, and since the optimal regions become equal to the Voronoi regions of these, we see that the VQ in the digital part approaches a VQ designed for a noiseless digital channel.

\footnotetext{
${ }^{5}$ The proof provided in the Appendix assumes that the noise in the digital part is nonzero, i.e., $P(j \mid i) \geq \varepsilon>0$ for all $j$ and $i$.
}

Input data: A fixed SNR $1 / \sigma^{2}$ (assumed equal in the digital and analog channels), and the corresponding transition probabilities $P(j \mid i)$ of the digital channel.

(0) Initialize the encoder regions $\left\{\mathcal{S}_{i}\right\}$, and set $\gamma=1 /\left(1+\sigma^{2}\right)$.

(i) Estimate the encoder centroids $\left\{\mathbf{m}_{x}(i)\right\}$, and the probabilities $\{P(i)\}$. Compute the vectors $\{\tilde{\mathbf{x}}(j)\}$ using (19). Determine $\left\{\mathbf{y}_{j}\right\}$, using (22), and then set $\left\{z_{i}\right\}$ according to $(17)$.

(ii) Estimate the power $P=E\left\|\mathbf{X}-\mathbf{z}_{J}\right\|^{2}$ of the residual in the analog part, and set $\alpha=\sqrt{d / P}$. Then let $\beta=\gamma / \alpha$ (c.f., (13)).

(iv) Compute $\left\{g_{i}\right\}$ using (24), and then redefine the encoder regions according to (25) (noting that we can use $\mathbf{m}_{y}(i)=\mathbf{z}_{i}$ where $\left\{\mathbf{z}_{i}\right\}$ were computed in (i)).

(v) Estimate the power $P=E\left\|\mathbf{X}-\mathbf{z}_{i}\right\|^{2}$, and set $\alpha=\sqrt{d / P}$. Set $\beta=\gamma / \alpha$.

(vi) Repeat from (i) until convergence.

Fig. 5. Description of design algorithm.

(VQ design for noiseless channels is described, e.g., in [27].)

- High noise level in the digital part: For $\{P(j \mid i)\}$ derived from a BSC, as in (1), increasingly high noise level in the digital channel corresponds to $q \rightarrow 1 / 2$. This will give

$$
P(j \mid i) \rightarrow(1 / 2)^{L}=1 / N, \quad \text { for all } j \text { and } i .
$$

First, noting that this implies $P(i \mid j) \rightarrow P(i), \forall j$, and then studying (19) we see that $\tilde{\boldsymbol{x}}(j) \rightarrow E[\boldsymbol{X}], \forall j$. Then, in solving (17) and (18), noting that we assume $\gamma \neq 1$, and using $\tilde{\boldsymbol{x}}(j)=E[\boldsymbol{X}]$, we get $\boldsymbol{z}_{i}=E[\boldsymbol{X}]$ and $\boldsymbol{y}_{j}=\tilde{\boldsymbol{x}}(j)=$ $E[\boldsymbol{X}]$, for all $i$ and $j$. Studying the expression (25) for the optimal encoder regions we see that since $g_{i} \rightarrow 0, \forall i$, and since $m_{y}(i) \rightarrow E[\boldsymbol{X}], \forall i$, there will, furthermore, only be one nonempty region, say $\mathcal{S}_{0}$. That is, we get a situation similar to the one obtained for $\gamma \rightarrow 1$, as studied above, where all codevectors become equal and where increasingly many encoder regions become empty until, finally, only one index is transmitted, irrespective of the input $\boldsymbol{x}$, and the decoder produces the expected value $E[\boldsymbol{X}]$ of the source for all received indexes.

\section{Training Algorithm}

The results presented in Section IV-A and -B can be utilized to formulate an iterative training algorithm for the HDA system. This algorithm is summarized in Fig. 5. With reference to this figure, the following is a list of comments to the design.

- Besides the source vector dimension $d$ and the size $N=2^{L}$ of the VQ (and assuming that the statistics of the source, as described by the pdf $p(\boldsymbol{x})$, are known), the algorithm takes as input the transition probabilities $\{P(j \mid i)\}$ of the digital channel and the SNR $1 / \sigma^{2}$ in the analog part. Assuming that the digital channel is derived from a binary Gaussian channel, the transition probabilities can be obtained from (1), (2) for a given noise variance $\sigma^{2}$.

- In Step 0) of the algorithm, the encoder regions can be initialized by using the Voronoi regions of a VQ trained for a noiseless channel and the source under consideration. Another alternative is to use the encoder of a COVQ trained for the digital channel $\{P(j \mid i)\}$.

- As mentioned earlier, an alternative to using (22) in step i) is to employ an iterative approach. In the systems inves- 
tigated in Section $\mathrm{V}$, we used this approach when training for a high SNR (and hence a $\gamma$ close to one), since in this case the matrix $\left(\boldsymbol{I}_{N}-\gamma \boldsymbol{F}\right)$ is close-to-singular and solving the equation system becomes numerically unstable. ${ }^{6}$

- Convergence, in step vi), may be checked by monitoring the distortion $D$, and stop the iterations when the relative improvement is small enough.

- Finally, it should be noted that the scaling constant $\alpha$ is updated in steps ii) and v) of the training and, strictly speaking, there is therefore no guarantee that the power constraint is satisfied at all instants of the algorithm (while our derivation assumed this). Consequently, convergence is not guaranteed. However, all our practical experience with implementing the training algorithm suggests that this issue is not a problem in practice, since in all cases we have encountered, the algorithm does converge to a stable solution.

\section{PERFORMANCE OF OPTIMIZED HDA SYSTEMS}

In this section, we evaluate the performance of our optimized VQ-based HDA system for the compression and transmission of two (unit variance) sources: a memoryless (i.i.d.) Gaussian source and a Gauss-Markov source with correlation parameter 0.9 . Motivated by a broadcast scenario, we use the design algorithm described in Fig. 5 to implement a fixed encoder, adaptive decoder (FEAD) optimized HDA system. More precisely, the proposed scheme, denoted by HDA-F $F_{*} E A D$, consists of an optimized HDA system in which the encoder (i.e., the parameters $\left\{\mathcal{S}_{i}\right\},\left\{\boldsymbol{z}_{i}\right\}$, and $\alpha$, where $\alpha$ is chosen to satisfy the power constraint) is designed for a fixed value $*$ (in decibels) of the channel SNR, $1 / \sigma^{2}$, and is not modified as the true SNR changes, while the decoder (i.e., the parameters $\left\{\boldsymbol{y}_{j}\right\}$ and $\beta$ ) has knowledge of the true SNR and thus adapts to it.

We present simulation results for different optimized HDA$\mathrm{F}_{*} \mathrm{EAD}$ schemes, and compare them with the basic (unoptimized) HDA-VQ system (discussed in Section III), a purely analog system, and several purely digital systems. All considered systems have an overall transmission rate of $r=2$ channel uses per source sample. We employed 2 million training vectors in designing codes with $d=8$ and $L=8$, and 8 million training vectors for VQ codes with $d=8$ and $L=16$; we used 500000 test vectors for the simulations. The training of each HDA system was initialized by using the encoder regions of a COVQ trained for the corresponding digital channel (see the remark concerning step 0) of the algorithm in Section IV-D). In Figs. 6-10, we show performance results in terms of the SDR for an i.i.d. Gaussian source (Figs. 6 and 8) and a Gauss-Markov source (Figs. 7, 9, and 10) transmitted over an AWGN channel via the following systems.

\footnotetext{
${ }^{6}$ This is the approach we employed, for high values of $\gamma$, to obtain the results of Section V. Another alternative, perhaps a preferred one, would be to use a more sophisticated technique tailored to solving close-to-singular equation systems [28].
}

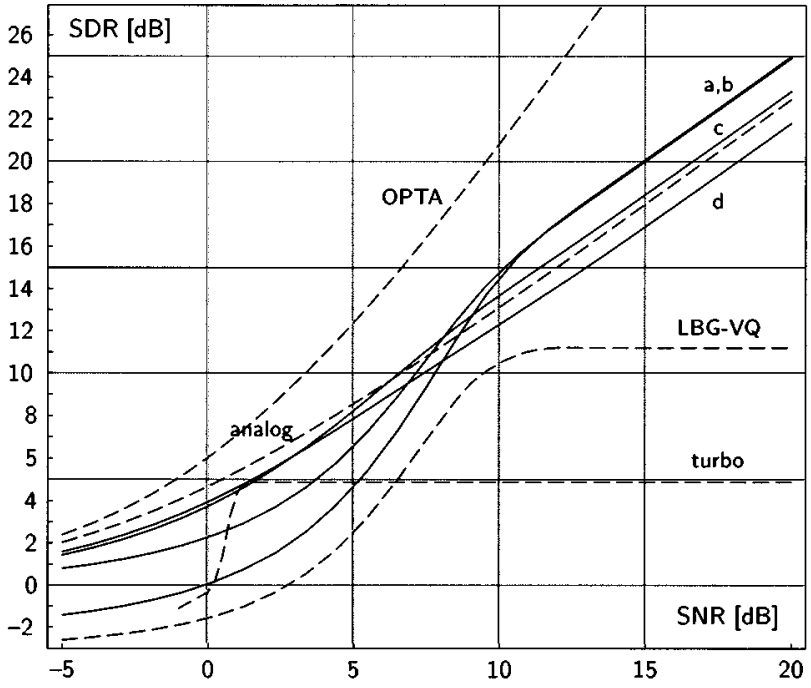

Fig. 6. i.i.d. Gaussian source. Solid lines from above at SNR $=15 \mathrm{~dB}$ : (a) HDA-VQ, and HDA-F $F_{*}$ EAD with “*” equal to (b) $10 \mathrm{~dB}$, (c) $5 \mathrm{~dB}$, (d) $0 \mathrm{~dB}$. Dashed lines from above at SNR $=15 \mathrm{~dB}$ : OPTA (unrestricted AWGN channel), purely analog, purely digital LBG-VQ ( $d=8$ and rate two), LBG-VQ-turbo ( $d=8$ and overall rate two).

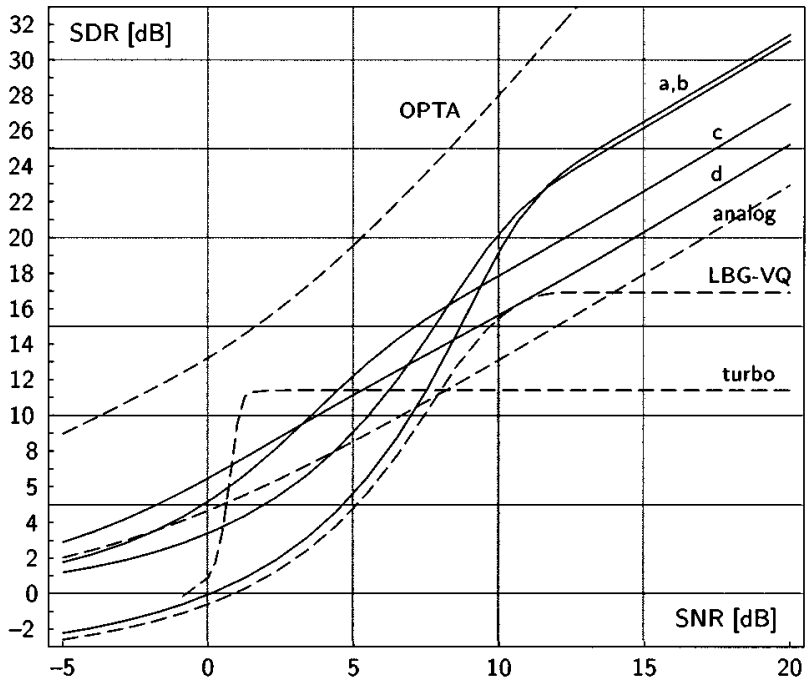

Fig. 7. Gauss-Markov source. Solid lines from above at $\mathrm{SNR}=15 \mathrm{~dB}$ : (a) HDA-VQ, and HDA-F ${ }_{*}$ EAD with “*” equal to (b) $10 \mathrm{~dB}$, (c) $5 \mathrm{~dB}$, (d) $0 \mathrm{~dB}$. Dashed lines from above at $\mathrm{SNR}=15 \mathrm{~dB}$ : OPTA (unrestricted AWGN channel), purely analog, purely digital LBG-VQ ( $d=8$ and rate two), LBG-VQ-turbo $(d=8$ and overall rate two $)$.

- Three (optimized) HDA-F $F_{*}$ EAD schemes shown in Figs. 6-9: HDA-F $\mathrm{F}_{0}$ EAD, HDA-F $\mathrm{F}_{5} \mathrm{EAD}$, and HDA- $\mathrm{F}_{10}$ EAD trained at an SNR of 0,5 , and $10 \mathrm{~dB}$, respectively. The HDA-F $F_{*}$ EAD schemes employ a quantizer with $d=8$ and a rate of $1 \mathrm{bit} /$ source sample $(L=8)$.

Note that the above HDA-F*EAD schemes assumed a binary-input binary-output channel in the digital part, since the underlying BPSK-modulated AWGN channel was assumed to be used with hard-decision demodulation. In Fig. 10, we present HDA-F $F_{*}$ EAD schemes that exploit the soft channel information in the digital part. To accommodate this change, the design algorithm was modified as 


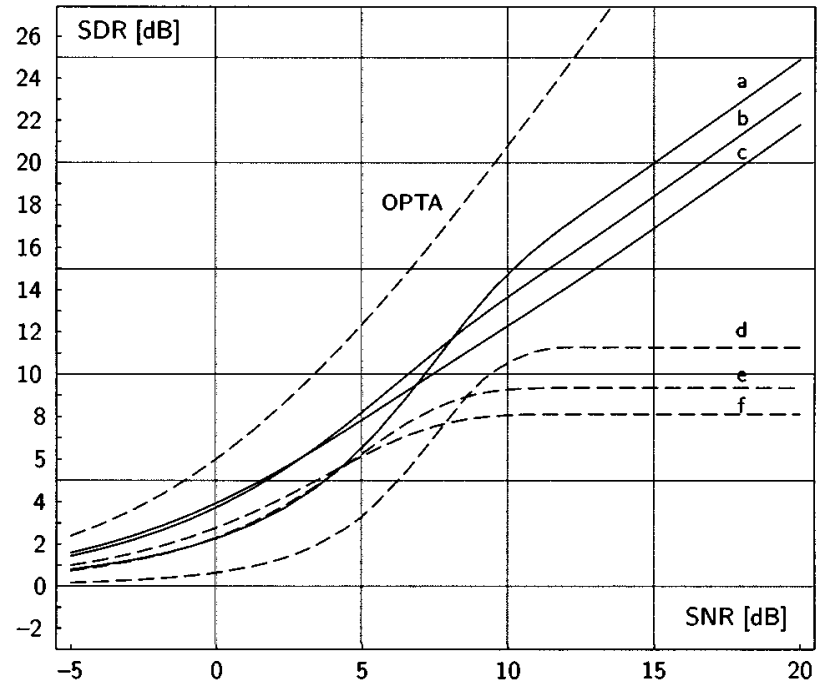

Fig. 8. i.i.d. Gaussian source. Solid lines from above at $\mathrm{SNR}=15 \mathrm{~dB}$ : HDA-F $F_{*}$ EAD with “*” equal to (a) $10 \mathrm{~dB}$, (b) $5 \mathrm{~dB}$, (c) $0 \mathrm{~dB}$. Dashed lines from above at SNR $=15 \mathrm{~dB}$ : OPTA (unrestricted AWGN channel), COVQ- ${ }_{\star}$ EAD with "*” equal to (d) $10 \mathrm{~dB}$, (e) $5 \mathrm{~dB}$, (f) $0 \mathrm{~dB}$. (The COVQs have $d=8$ and rate two, with encoder optimized at $\mathrm{SNR} *$ and with adaptive decoding.)

follows. Assume that the vector $R \in \mathbb{R}^{L}$ is received at the output of the underlying Gaussian channel of the digital part as a result of transmitting $L$ bits of an index $i$. Soft decoding was applied (cf., [29], [26], [11], [30]) in the sense that $\tilde{\boldsymbol{x}}(j)$ and $\tilde{\boldsymbol{z}}(j)$, as defined in (16), are replaced with the estimators $E[X \mid \boldsymbol{R}=\boldsymbol{r}]$ and $E\left[\boldsymbol{z}_{I} \mid \boldsymbol{R}=\boldsymbol{r}\right]$, respectively, in the decoding of the digital part (see, e.g., [26] for more details regarding the implementation of these estimators). Thus, instead of performing a table lookup decoding based on the decoder codevectors $\left\{\boldsymbol{y}_{j}\right\}$, the decoder of the digital part uses the mapping

$$
\boldsymbol{y}(\boldsymbol{r}) \triangleq(1-\gamma) E[\boldsymbol{X} \mid \boldsymbol{R}=\boldsymbol{r}]+\gamma E\left[\boldsymbol{z}_{I} \mid \boldsymbol{R}=\boldsymbol{r}\right]
$$

based on the received soft data $\boldsymbol{R}=\boldsymbol{r}$. This results in an overall receiver output given by

$$
\hat{\boldsymbol{x}}=\hat{\boldsymbol{x}}(\boldsymbol{r}, \boldsymbol{u})=\boldsymbol{y}(\boldsymbol{r})+\beta \boldsymbol{u} .
$$

- The basic (unoptimized) HDA-VQ scheme shown in Figs. 6 and 7. It is designed with $\beta$ given by (13) and using the LBG algorithm [21] for the digital part with $d=8$ and source coding rate of $1 \mathrm{bit} /$ source sample $(L=8)$. (The codebook of the resulting VQ then specifies both $\left\{\boldsymbol{z}_{i}\right\}$ and $\left\{\boldsymbol{y}_{j}\right\}$, and the encoder regions are given by the Voronoi regions of the VQ codebook; cf., Section III.)

- A purely analog system employing an LMMSE decoder (cf., Section III above). The performance of this system is shown in Figs. 6 and 7.

- Three purely digital systems: two tandem source-channel coding systems (LBG-VQ and turbo) and one joint sourcechannel coding system (COVQ- $\left.F_{*} E A D\right)$. These systems are described as follows.

$L B G-V Q$ : It is a basic VQ with $d=8$ and rate $2(L=$ $16)$, designed for the noiseless digital channel using

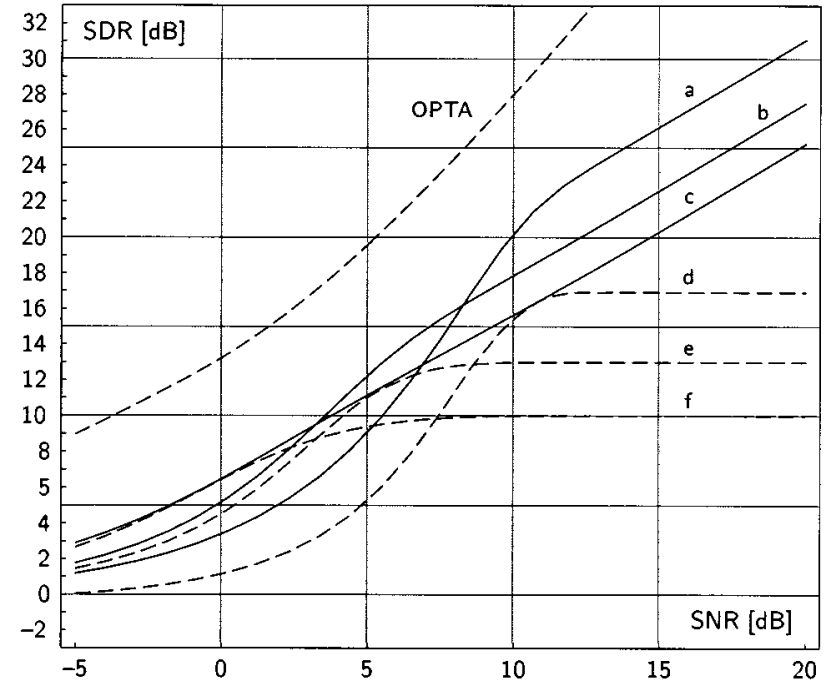

Fig. 9. Gauss-Markov source. Solid lines from above at SNR $=15 \mathrm{~dB}$ HDA- $F_{*}$ EAD with "*” equal to (a) $10 \mathrm{~dB}$, (b) $5 \mathrm{~dB}$, (c) $0 \mathrm{~dB}$. Dashed lines from above at $\mathrm{SNR}=15 \mathrm{~dB}$ : OPTA, COVQ-F ${ }_{*} \mathrm{EAD}$ with “*” equal to (d) 10 $\mathrm{dB}$, (e) $5 \mathrm{~dB}$, (f) $0 \mathrm{~dB}$. (The COVQs have $d=8$ and rate two, with encoder optimized at SNR $*$ and with adaptive decoding.)

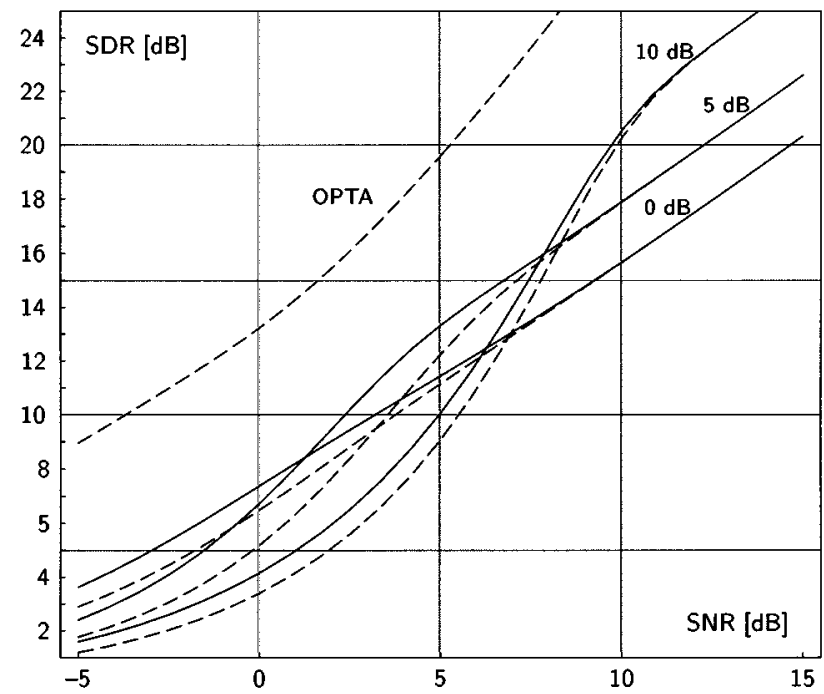

Fig. 10. Gauss-Markov source. Solid lines from above at $\mathrm{SNR}=10 \mathrm{~dB}$ HDA- $F_{*}$ EAD with $*=10,5,0[\mathrm{~dB}]$ using soft decoding in the digital part. Dashed lines from above at SNR $=10 \mathrm{~dB}$ : OPTA, and HDA-F $\mathrm{F}_{*} \mathrm{EAD}$ with $*=10,5,0[\mathrm{~dB}]$ using hard decoding in the digital part.

the LBG-VQ algorithm (the same code as also used in Section III). It is shown in Figs. 6 and 7.

- $\quad L B G-V Q$-turbo: It consists of an eight-dimensional LBG-VQ with a rate of $1 \mathrm{bit} /$ source sample, followed by a rate-1/2, $(n, k)=(2048,1024)$ turbo code [23] with generators $(37,21)$. (Shown in Figs. 6 and 7; the same system as in Section III.)

- $\quad C O V Q-F_{*} E A D$ : It consists of a COVQ with $d=8$ and rate $2(L=16)$, with the encoder optimized at $\mathrm{SNR} *$ and adaptive decoding (as in $\mathrm{HDA}-\mathrm{F}_{*} \mathrm{EAD}$ ). It is shown in Figs. 8 and 9. 
- The optimal performance theoretically attainable, as defined in (7) above (for an unrestricted AWGN channel in the digital part).

We observe from Figs. 6-10 that the HDA-based systems offer a robust and graceful performance over the entire range of the SNRs, with the HDA-FEAD systems employing soft decoding providing the best performance (see Fig. 10). More specifically, the HDA-FEAD systems perform very well in the vicinity of the SNR at which their encoder was designed; they also provide a smooth degradation/improvement as the true SNR varies away from the designed SNR. We remark that the optimized HDA-FEAD systems provide substantial improvements over the basic HDA-VQ scheme for low to medium SNRs. For high SNRs, the HDA-F ${ }_{10}$ EAD scheme nearly matches the performance of the HDA-VQ system (Figs. 6 and $7)$. We also note that all HDA-FEAD systems outperform the analog (cf., Figs. 6 and 7) for most SNRs. The gains over the analog systems are more pronounced for the Gauss-Markov source; this is expected, since, unlike the HDA schemes, the analog system fails to exploit the source memory. Furthermore, the HDA-FEAD systems provide considerable gains over all purely digital systems (cf., Figs. 6-9) for most SNRs. We also observe that the use of soft channel information in the design of the digital part significantly enhances the performance of the HDA-FEAD systems, particularly at low SNRs (see Fig. 10).

Finally, before closing this section, we remark that while the theory of Sections II and III is general in the sense that most of the treatment holds for a general set of transition probabilities $\{P(j \mid i)\}$, and hence any (memoryless) modulation constellation in the digital part (cf., footnote 2 ), we have chosen to carry out all simulations for binary modulation. The main reason for this is again our objective of keeping the system simple and robust. We also note that increasing the rate in the digital transmission (the rate can be increased by choosing a larger modulation signal set) makes the digital part "more analog" and this is essentially not a desired feature of the system, since the purpose of the analog part is to take care of the residual error, at high SNRs, due to the low rate in the digital part. Hence, with simplicity and robustness in mind, binary modulation (BPSK) is a natural choice since it is a simple modulation format that works comparatively well at low SNRs. While both the power and rate allocation problems are interesting in their own rights, we have chosen not to study them in detail. Optimizing the power and/or rate allocation between the digital and analog parts will not significantly change the overall (global) system behavior. Furthermore, robustness will be lost since the more parameters (in the transmitter) are tailored to a specific design SNR, the less robust will the system be when the true SNR deviates from it.

\section{SUMmARY AND CONCLUSION}

In this work, a VQ-based HDA joint source-channel coding system for AWGN channels was investigated. This HDA system, which exploits the attributes of both purely analog systems and purely digital joint source-channel coding systems, was optimized via an iterative design algorithm that minimizes, for each SNR, the end-to-end mean square-error distortion subject to a power constraint in the analog part of the system. The behavior of the system was also analyzed for various asymptotic conditions on the noise level in the analog and digital parts.

The performance of optimized HDA systems with a fixed encoder and an adaptive decoder (FEAD) was assessed for a wide range of channel conditions; comparisons were also made with the unoptimized HDA system, a purely analog system, and various (tandem and joint source-channel coding) purely digital systems. Optimized HDA-FEAD schemes which exploit the soft channel information in the digital part were also implemented. Simulation results for memoryless Gaussian as well as Gauss-Markov sources demonstrated a very robust and graceful performance of the HDA systems over the entire range of the channel SNRs. Significant coding gains were also achieved over the unoptimized HDA system and the purely analog and digital systems.

The system investigated in this paper only works for channel transmission rates above one channel use per source sample. An important topic for further study is to investigate how the system can be modified to work at rates below one. One strong candidate system to study for this purpose is the "dual system" presented by Mittal and Phamdo in [12]. Preliminary results in this direction are reported in [31].

\section{APPENDIX}

Here we investigate the properties of the matrix $G \triangleq I_{N}-\gamma \boldsymbol{F}$ in some more detail. This matrix was defined in connection with (20) and its properties are important, for example, in analyzing under what conditions the equation system (20) has a unique solution.

The matrix $G$ is defined in terms of the real parameter $\gamma$ and the matrix $\boldsymbol{F}$, with elements $(\boldsymbol{F})_{j k}$ as given by (21). To begin with, we note that since $\sum_{j}(\boldsymbol{F})_{j k}=1, \forall k$, and since $(\boldsymbol{F})_{j k} \geq$ $0, \boldsymbol{F}$ is a column stochastic matrix. Hence, $\boldsymbol{F}$ has the real number 1 as eigenvalue, and all other eigenvalues have modulus less than or equal to 1 . To prove this statement, let $\boldsymbol{A}$ be an arbitrary square matrix with nonnegative elements, and assume that $\boldsymbol{A}$ has eigenvalues $\left\{\lambda_{l}\right\}$. Furthermore, let $\rho(\boldsymbol{A})=\max _{l}\left|\lambda_{l}\right|$. Then, according to [32, Corollary 8.1.30], if $\boldsymbol{x}$ is an eigenvector of $A$ with all elements positive, $\boldsymbol{x}>0$, then the corresponding eigenvalue is $\rho(A)$. Consequently, letting $\boldsymbol{e}$ be a vector with all elements equal to 1 and noting that $e$ is an eigenvector to $F^{T}$, with its corresponding eigenvalue being the real number 1 , we know that $\rho\left(\boldsymbol{F}^{T}\right)=1$. Moreover, since a square matrix and its transpose have the same eigenvalues, we also have that $\rho(\boldsymbol{F})=1$, hence proving the desired result. Thus, knowing that all eigenvalues of $\boldsymbol{F}$ are less than or equal to 1 in modulus, we get the result that the eigenvalues of the matrix $\boldsymbol{G}$ are all nonzero as long as $\gamma<1$ (since the eigenvalues of $\boldsymbol{G}$ are obtained as $1-\gamma$ times the eigenvalues of $\boldsymbol{F}$ ). This proves that $\boldsymbol{G}$ is invertible for all $0 \leq \gamma<1$

In Section IV-C, we studied the case of an arbitrary set of transition probabilities $\{P(j \mid i)\}$ and $\gamma \rightarrow 1$. Assuming that $\{P(j \mid i)\}$ are derived from a binary symmetric channel, as in 
(1), all transition probabilities are positive as long as $0<q<1$. Consequently, assuming $0<q<1$ in the digital part we have $P(j \mid i)>0$ and hence, using the definition (21), it follows that

$$
\begin{aligned}
(\boldsymbol{F})_{j k} & =\sum_{i} \operatorname{Pr}(I=i \mid J=k) \operatorname{Pr}(J=j \mid I=i) \\
& \geq \min _{i}\{P(j \mid i)\} \sum_{i} \operatorname{Pr}(I=i \mid J=k) \\
& =\min _{i}\{P(j \mid i)\}>0
\end{aligned}
$$

for all $j$ and $k$. Hence, when $\{P(j \mid i)\}$ are derived from a BSC with positive crossover probability $q$ the matrix $\boldsymbol{F}$ is strictly positive. This means that by Perron's theorem [32, p. 500] $F$ has a unique eigenvalue of maximum modulus, and since $\boldsymbol{F}$ is stochastic we know that this eigenvalue is the real number 1 . That is, $\boldsymbol{F}$ has one eigenvalue equal to 1 and all other eigenvalues of $\boldsymbol{F}$ are less than 1 in modulus. Now we return to the system (22), and study it under the assumption $P(j \mid i)>0$. Taking transpose of both sides in (22), we get

$$
\left(\boldsymbol{I}_{N}-\gamma \boldsymbol{F}^{T}\right) \boldsymbol{Y}^{T}=(1-\gamma) \tilde{\boldsymbol{X}}^{T}
$$

In calculating a Schur decomposition of $\boldsymbol{F}^{T}$ (cf., [32, Theorem 2.3.1]) we get $\boldsymbol{F}^{T}=\boldsymbol{U} \boldsymbol{T} \boldsymbol{U}^{H}$ (where $[\cdot]^{H}$ denotes the Hermitian transpose, defined as $\boldsymbol{U}^{H}=(\overline{\boldsymbol{U}})^{T}$ with $\overline{\boldsymbol{U}}$ being the component-wise complex conjugate of $\boldsymbol{U}$ ) where $\boldsymbol{U}$ is unitary (i.e., $\left.\boldsymbol{U} \boldsymbol{U}^{H}=\boldsymbol{I}_{N}\right)$ and where $\boldsymbol{T}$ is an upper triangular matrix with the eigenvalues of $\boldsymbol{F}^{T}$ on its diagonal. The decomposition can always be chosen such that the unique largest eigenvalue (in modulus), the number 1, appears in the uppermost left position of $T$, and with the corresponding (normalized) eigenvector $N^{-1 / 2} \boldsymbol{e}$ (where $e$ is the all-one vector of size $N$ ) in the first column of $\boldsymbol{U}$. Then, using $\boldsymbol{F}^{T}=\boldsymbol{U} \boldsymbol{T} \boldsymbol{U}^{H}$ we get (for all $0 \leq \gamma<1$ )

$$
\boldsymbol{Y}^{T}=\boldsymbol{U}\left[(1-\gamma)\left(\boldsymbol{I}_{N}-\gamma \boldsymbol{T}\right)^{-1}\right] \boldsymbol{U}^{H} \tilde{\boldsymbol{X}}^{T} .
$$

Now, in partitioning $\boldsymbol{T}$ according to

$$
\boldsymbol{T}=\left[\begin{array}{cc}
1 & \mathbf{b}^{T} \\
\mathbf{0} & \boldsymbol{B}
\end{array}\right]
$$

where $\boldsymbol{b}$ is a column vector, $\mathbf{0}$ is the all-zero column vector of size $N-1$, and $\boldsymbol{B}$ is an upper triangular matrix with the $N-$ 1 remaining eigenvalues of $F^{T}$ on its diagonal, we get (still assuming $0 \leq \gamma<1$ )

$$
(1-\gamma)\left(\boldsymbol{I}_{N}-\gamma \boldsymbol{T}\right)^{-1}=\left[\begin{array}{cc}
1 & \gamma \boldsymbol{b}^{T}\left(\boldsymbol{I}_{N-1}-\gamma \boldsymbol{B}\right)^{-1} \\
\mathbf{0} & (1-\gamma)\left(\boldsymbol{I}_{N-1}-\gamma \boldsymbol{B}\right)^{-1}
\end{array}\right]
$$

(this result can easily be verified). Here we note that since $B$ is an upper triangular matrix with all diagonal entries less than 1 (in modulus), the inverse $\left(\boldsymbol{I}_{N-1}-\gamma \boldsymbol{B}\right)^{-1}$ exists for all $0 \leq$ $\gamma \leq 1$ (i.e., including $\gamma=1$ ). Hence, studying (28) we see that as $\gamma \rightarrow 1$ the solution for $Y^{T}$ in (27) has a well-defined limit, namely

$$
\begin{aligned}
\boldsymbol{Y}^{T} & \rightarrow \boldsymbol{U}\left[\begin{array}{cc}
1 & \boldsymbol{b}^{T}\left(\boldsymbol{I}_{N-1}-\boldsymbol{B}\right)^{-1} \\
\mathbf{0} & \mathbf{0 0}^{T}
\end{array}\right] \boldsymbol{U}^{H} \tilde{\boldsymbol{X}}^{T} \\
& =N^{-1 / 2} \boldsymbol{e} \boldsymbol{e}^{T} \boldsymbol{D} \boldsymbol{U}^{H} \tilde{\boldsymbol{X}}^{T}
\end{aligned}
$$

where $D$ is a diagonal matrix with the elements of the row vector $\left[1 \boldsymbol{b}^{T}\left(\boldsymbol{I}_{N-1}-\boldsymbol{B}\right)^{-1}\right]$ on its diagonal, and where we used the fact that the first column of $\boldsymbol{U}$ is $N^{-1 / 2} \boldsymbol{e}$. This result shows, for example, that as $\gamma \rightarrow 1$ the matrix $\boldsymbol{Y}$ approaches a matrix with all columns $\boldsymbol{y}_{j}$ equal to the single vector $\boldsymbol{y}_{0}=N^{-1 / 2} \tilde{\boldsymbol{X}} \overline{\boldsymbol{U}} \boldsymbol{D} \boldsymbol{e}$.

\section{ACKNOWLEDGMENT}

The authors wish to thank the anonymous reviewers for numerous comments that helped improve the quality of this paper.

\section{REFERENCES}

[1] M. Skoglund, N. Phamdo, and F. Alajaji, "VQ-based hybrid digital-analog joint source-channel coding," in Proc. IEEE Int. Symp. Information Theory, Sorrento, Italy, June 2000, p. 403.

[2] T. M. Cover and J. A. Thomas, Elements of Information Theory. New York: Wiley, 1991.

[3] A. Kurtenbach and P. Wintz, "Quantizing for noisy channels," IEEE Trans. Commun. Technol., vol. COM-17, pp. 291-302, Apr. 1969.

[4] H. Kumazawa, M. Kasahara, and T. Namekawa, "A construction of vector quantizers for noisy channels," Electron. and Engineering in Japan, vol. 67-B, pp. 39-47, Jan. 1984.

[5] K. A. Zeger and A. Gersho, "Vector quantizer design for memoryless noisy channels," in Proc. IEEE Int. Conf. Commun. (ICC), Philadelphia, PA, 1988, pp. 1593-1597.

[6] N. Farvardin, "A study of vector quantization for noisy channels," IEEE Trans. Inform. Theory, vol. 36, pp. 799-809, July 1990.

[7] N. Farvardin and V. Vaishampayan, "On the performance and complexity of channel-optimized vector quantizers," IEEE Trans. Inform. Theory, vol. 37, pp. 155-159, Jan. 1991.

[8] K. A. Zeger and A. Gersho, "Pseudo-Gray coding," IEEE Trans. Commun., vol. 38, pp. 2147-2158, Dec. 1990.

[9] F. Alajaji, N. Phamdo, and T. Fuja, "Channel codes that exploit the residual redundancy in CELP-encoded speech," IEEE Trans. Speech Audio Processing, vol. 4, pp. 325-336, Sept. 1996.

[10] J. Kroll and N. Phamdo, "Analysis and design of trellis codes optimized for a binary symmetric Markov source with MAP detection," IEEE Trans. Inform. Theory, vol. 44, pp. 2977-2987, Nov. 1998.

[11] M. Skoglund, "Soft decoding for vector quantization over noisy channels with memory," IEEE Trans. Inform. Theory, vol. 45, pp. 1293-1307, May 1999.

[12] U. Mittal and N. Phamdo, "Hybrid digital-analog (HDA) joint source-channel codes for broadcasting and robust communications," IEEE Trans. Inform. Theory, submitted for publication.

[13] - "Nearly robust joint source-channel codes," in Proc. Canadian Workshop on Information Theory, Kingston, ON, Canada, June 1999, pp. 63-66.

[14] N. Phamdo and U. Mittal, "Joint source-channel speech coder using hybrid digital-analog (HDA) modulation," IEEE Trans. Speech Audio Processing, submitted for publication.

[15] W. F. Schreiber, "Advanced television systems for terrestrial broadcasting: Some problems and some proposed solutions," Proc. IEEE, vol. 83, pp. 958-981, June 1995.

[16] S. Shamai (Shitz), S. Verdú, and R. Zamir, "Systematic lossy source/channel coding," IEEE Trans. Inform. Theory, vol. 44, pp. 564-579, Mar. 1998.

[17] J. M. Lervik, A. Grovlen, and T. Ramstad, "Robust digital signal compression and modulation exploiting the advantages of analog communications," in Proc. IEEE Global Telecommunications Conf. (GLOBECOM), Singapore, Nov. 1995, pp. 1044-1048.

[18] A. Fuldseth and T. A. Ramstad, "Bandwidth compression for continuous amplitude channels based on vector approximation to a continuous subset of the source signal space," in Proc. IEEE Int. Conf. Acoustics, Speech and Signal Processing, Munich, Germany, June 1997, pp. 3093-3096.

[19] I. Kozintsev and K. Ramchandran, "Hybrid compressed-uncompressed framework for wireless image transmission," in Proc. IEEE Int. Conf. Communications, Montréal, QC, Canada, June 1997, pp. 77-80.

[20] H. C. Papadopoulos and C.-E. W. Sundberg, "Simultaneous broadcasting of analog FM and digital audio signals by means of adaptive precanceling techniques," IEEE Trans. Commun., vol. 46, pp. 12331242, Sept. 1998. 
[21] Y. Linde, A. Buzo, and R. M. Gray, "An algorithm for vector quantizer design," IEEE Trans. Commun., vol. 28, pp. 84-95, Jan. 1980.

[22] T. Berger, Rate Distortion Theory-A Mathematical Basis for Data Compression. Englewood Cliffs, NJ: Prentice-Hall, 1971.

[23] C. Berrou and A. Glavieux, "Near optimum error correcting coding and decoding: Turbo-codes," IEEE Trans. Commun., vol. 44, pp. 1261-1271, Oct. 1996

[24] N. Phamdo, N. Farvardin, and T. Moriya, "A unified approach to treestructured and multistage vector quantization for noisy channels," IEEE Trans. Inform. Theory, vol. 39, pp. 835-850, May 1993.

[25] P. Hedelin, P. Knagenhjelm, and M. Skoglund, "Theory for transmission of vector quantization data," in Speech Coding and Synthesis, W. B. Kleijn and K. K. Paliwal, Eds. Amsterdam, The Netherlands: Elsevier, 1995, ch. 10, pp. 347-396.

[26] M. Skoglund and P. Hedelin, "Hadamard-based soft decoding for vector quantization over noisy channels," IEEE Trans. Inform. Theory, vol. 45, pp. 515-532, Mar. 1999.
[27] A. Gersho and R. M. Gray, Vector Quantization and Signal Compression. Dordrecht, The Netherlands: Kluwer, 1992.

[28] G. H. Golub and C. F. Van Loan, Matrix Computations, 3rd ed. Baltimore, MD: Johns Hopkins Univ. Press, 1996.

[29] V. Vaishampayan and N. Farvardin, "Joint design of block source codes and modulation signal sets," IEEE Trans. Inform. Theory, vol. 38, pp. 1230-1248, July 1992.

[30] N. Phamdo and F. Alajaji, "Soft-decision demodulation design for COVQ over white, colored and ISI Gaussian channels," IEEE Trans. Commun., vol. 48, pp. 1499-1506, Sept. 2000.

[31] M. Skoglund, N. Phamdo, and F. Alajaji, "Hybrid digital-analog coding for bandwidth compression/expansion using VQ and turbo codes," in Proc. IEEE Int. Symp. Information Theory, Washington, DC, June 2001, p. 260.

[32] R. A. Horn and C. R. Johnson, Matrix Analysis. Cambridge, U.K.: Cambridge Univ. Press, 1985. 